\title{
Somatic mosaicism for a MECP2 mutation associated with classic Rett syndrome in a boy
}

\author{
Meral Topçu ${ }^{1}$, Cemaliye Akyerli ${ }^{2}$, Ayça Sayı $^{2}$, Gökçe A Törüner ${ }^{2}$, Süha R Koçoğlu ${ }^{2}$, \\ Mine Cimbiş ${ }^{1}$ and Tayfun Özçelik*,2 \\ ${ }^{1}$ Department of Pediatric Neurology, Hacettepe University School of Medicine, Sihhiye, Ankara 0600, Turkey; \\ ${ }^{2}$ Department of Molecular Biology and Genetics, Faculty of Science, Bilkent University, Ankara 06533, Turkey
}

Rett syndrome is a severe neurodevelopmental disorder that arises from mutations in the X-linked MECP2 gene. It is almost exclusively seen in girls due to the predominant occurrence of the mutations on the paternal X-chromosome, and also the early postnatal lethal effect of the disease causing mutations in hemizygous boys. We identified a boy with features of classic Rett syndrome who is mosaic for the truncating MECP2 mutation R270X. Chromosome analysis showed normal karyotype. These results indicate that a MECP2 mutation associated with Rett syndrome in females could lead to a similar phenotype in males as a result of somatic mosaicism.

European Journal of Human Genetics (2002) 10, 77-81. DOI: 10.1038/sj/ejhg/5200745

Keywords: Rett syndrome; MECP2; somatic mosaicism

\section{Introduction}

Classical Rett syndrome (RTT-MIM 312750) was first defined as a neurodevelopmental disorder that is primarily seen in girls. ${ }^{1,2}$ Following the hypothesis of X-linked dominant mutations that are lethal in hemizygous males, ${ }^{2}$ the RTT gene was localised to Xq28 by exclusion mapping studies in rare familial cases. ${ }^{3}$ Subsequently, MECP2 (methyl-CpGbinding protein 2) was identified as the gene responsible for this disorder by a systematic search for mutations in genes from the Xq28 region in RTT patients. ${ }^{4}$ Normal development until 6-18 months of age, followed by gradual loss of speech and purposeful hand use, microcephaly, seizures, ataxia, and stereotypic hand movements are among the cardinal features of Rett syndrome. ${ }^{5}$ Femaleness is also included in the abovementioned diagnostic criteria.

A wide range of MECP2 mutations has been identified in Rett syndrome patients. ${ }^{6}$ Interestingly, mutations of the

*Correspondence: T Özçelik, Department of Molecular Biology and Genetics, Faculty of Science, Bilkent University, 06533 Bilkent, Ankara, Turkey. Tel: +90 312266 5081; Fax: +90 3122665097 ;

E-mail: tozcelik@fen.bilkent.edu.tr

Received 12 July 2001; revised 16 October 2001; accepted 18 October 2001 same gene have been documented in male patients affected by severe encephalopathy, ${ }^{3,6}$ and X-linked mental retardation. ${ }^{7}$ One boy affected with a nonfatal neurodevelopmental disorder with similarities to Rett syndrome was found to be mosaic for a MECP2 gene mutation. ${ }^{8}$ In addition, an Angelman syndrome-like phenotype has recently been documented in girls with MECP2 mutations. ${ }^{9}$

We and others have observed boys with clinical features of Rett syndrome and a normal male karyotype. ${ }^{10-12}$ Several cases of 47,XXY males with a clinical diagnosis of Rett syndrome have been reported, although to our knowledge only one has been reported so far as having a MECP2 mutation. ${ }^{13}$ In order to determine whether MECP2 is involved in the development of the disease phenotype in males, we performed mutation screening in a patient not reported before, and diagnosed as Rett syndrome.

\section{Materials and methods}

Case report

BF was the product of a non-eventful pregnancy born to a 40year-old father and 29-year-old mother. He has two brothers (15 and 17 years old), and one sister (18 years old), who are all healthy. His birth weight was $3500 \mathrm{~g}$. Although he appeared 
to be a hypotonic child, he had normal psychomotor development through the first 6 months. Head control was achieved during the first 4 months. He sat with support at age 5 months, and without support at 12 months. He rolled over, transferred toys from one hand to the other, and babbled nonspecifically at around 6-8 months. He was also able to bring the milk bottle to his mouth alone. Loss of acquired purposeful hand skills began around 11 months, and stereotypic hand movements such as patting, squeezing became apparent at 15 months. He never crawled or walked. He has never spoken. None of the exclusion criteria (evidence of intrauterine growth retardation, organomegaly, retinopathy, microcephaly at birth etc.) was ever present. We first saw him at 2 years of age, and a tentative diagnosis of Rett syndrome was made. On examination at 12 years of age (Figure 1, Table 1), he was microcephalic with a head circumference of $47 \mathrm{~cm}$ (below second percentile), and

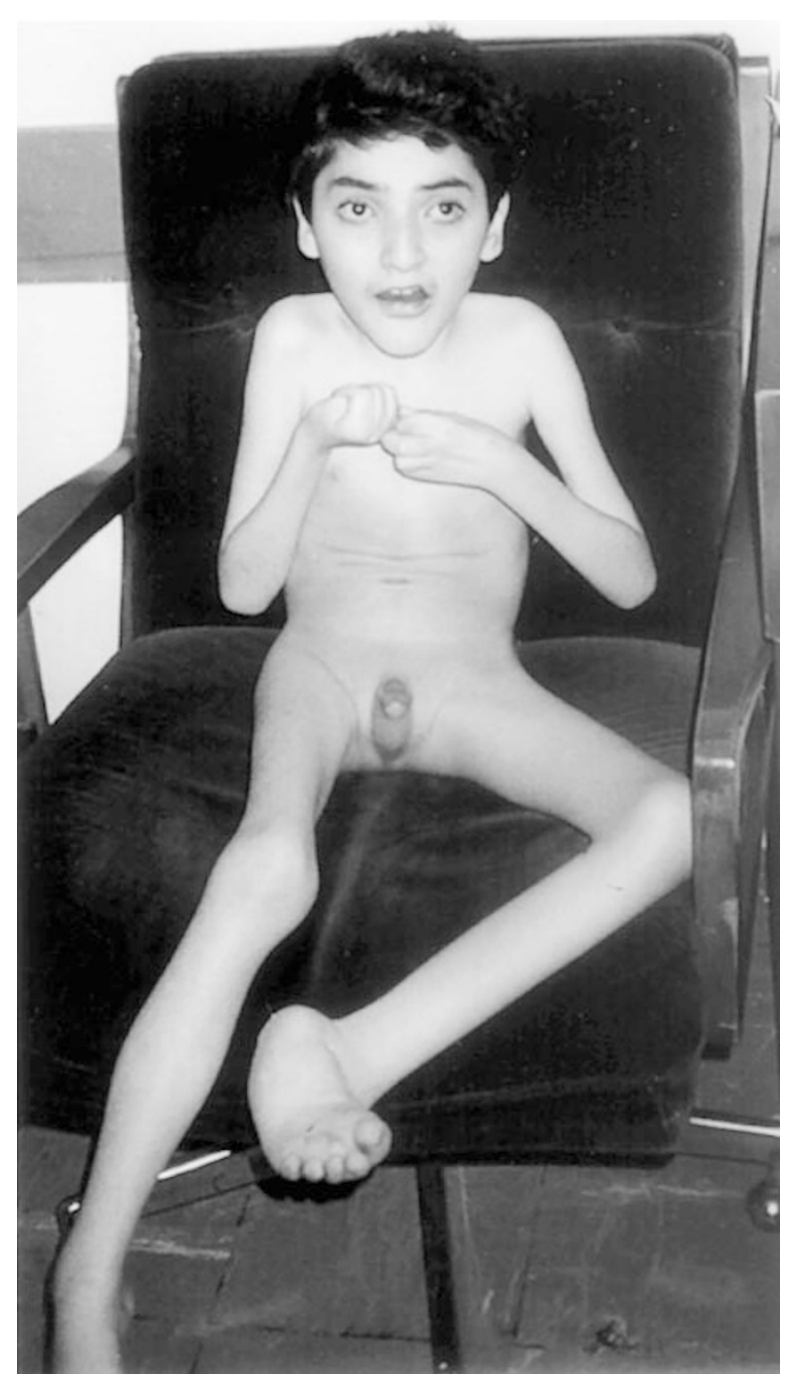

Figure 1 Photograph of BF at 12 years of age. brachycephalic with titubation of the head. His height was $110 \mathrm{~cm}$ and weight $18 \mathrm{~kg}$, which are both below the third percentile. Sialorrhea and bruxism were present. Stereotypic hand movements, tremors, and apraxia were noted. Deep tendon reflexes were increased bilaterally, and the clonus and Babinski signs were positive. His parents describe $\mathrm{BF}$ as a happy child who likes listening to music. No breath holding or abnormal skin pigmentation was observed. He had a thoracic scoliosis and poor lower-limb musculature. His hands and feet were small, and cold. Examination of the genitalia revealed presence of hypospadias and cryptorchidism. He experienced a total of three febrile seizures at the ages of five, 11, and 15 months, which was treated with phenobarbital for a short period of time. Currently he is not on this medication.

Electroencephalography showed excess of slow-wave activity and paroxysmal sharp theta wave activity prominent on wake recordings of frontal regions. A magnetic resonance scan at age 12 was normal. Based on these findings, RTT currently at stage IV was considered. Therefore, we obtained genomic DNA isolated from venous blood and hair root samples. Informed consent was obtained from each subject.

\section{Genotyping}

As a first step of the MECP2 gene screening procedure, we looked for eight recurrent mutations (R106W, P152R, T158M, R168X, R255X, R270X, R288X, and R306C) by PCR, and restriction enzyme digestion, ${ }^{4,6}$ which was further confirmed by sequencing. Two different sets of primers were used for the detection of the R270X mutation. The first set is 5'-GGC AGG AAG CGA AAA GCT GAG-3' and 5'-TGA GTG GTG GTG ATG GTG GTG GG-3' which yields a 366 bp fragment. The second set of primers which amplify a $380 \mathrm{bp}$ fragment was used to confirm the results: 5'AAC CAC CTA AGA AGC CAA A-3' and 5'-CTG CAC AGA TCG GAT AGA AGA C-3'. All reactions were performed in a total volume of $25 \mu \mathrm{l}$, with $10 \times$ buffer, $1.5 \mathrm{U}$ Taq polymerase, $1.5 \mathrm{~mm} \mathrm{MgCl}_{2}, 200 \mu \mathrm{M} \mathrm{dNTP}$, and $10 \mathrm{pmol}$ of each primer. PCR conditions were as follows: $95^{\circ} \mathrm{C}$ for $3 \mathrm{~min}, 30$ cycles of $30 \mathrm{~s}$ at $94^{\circ} \mathrm{C}, 58^{\circ} \mathrm{C}$ and $30 \mathrm{~s}$ at $72^{\circ} \mathrm{C}$. Ten minutes of $72^{\circ} \mathrm{C}$ was added at the extension step.

\section{Results}

We identified the truncating mutation $808 \mathrm{C} \rightarrow \mathrm{T}(\mathrm{R} 270 \mathrm{X})$ in BF's DNA sample (Figure 2A). The same mutation was present also in two girls diagnosed with RTT. ${ }^{14}$ Interestingly, the mutant allele in the affected boy was present along with the wild-type allele though at a reduced dosage when compared with the samples of the two girls. Whereas the mutant (T) and the wild-type (C) nucleotide peaks were equal in the two girls, the T allele peak was smaller in the boy. In order to quantitate the dosage of the alleles, genomic DNA samples of the boy obtained from three independent blood samplings done at different times, and from one of the girls was re-amplified with the same primers used in the sequencing reactions, and 
Table 1 Scoring for BF's different clinical features ${ }^{a}$

\begin{tabular}{ll}
\hline Clinical feature & Finding \\
\hline Head circumference during the first year & Normal at birth but decelerating \\
Developmental progress (birth to 12 months) & Suboptimal progress \\
Present head circumference & Below 3rd percentile \\
Present weight & Below 3rd percentile \\
Present height & Below 3rd percentile \\
Muscle tone & Severe hypertonia \\
Spine posture & Severe scoliosis \\
Joint contractures & Severe contractures \\
Gross motor function & Cannot walk with support \\
Hand stereotypy & Mild patting and squeezing \\
Other involuntary movements & Dominant dystonia \\
Voluntary hand use & None \\
Oro-motor difficulty & Delayed chewing, swallowing \\
Intellectual ability & Infant level \\
Speech & Currently uses no real words with meaning \\
Epilepsy & Previous epileptic seizures \\
Breathing & Normal rhythm \\
Peripheral circulation of extremities & Cold and discoloured with atrophic changes \\
Mood disturbance & Normal \\
Sleep disturbance & Normal \\
\hline
\end{tabular}

${ }^{\mathrm{a} A d a p t e d}$ from Kerr AM et al. $^{21}$

A

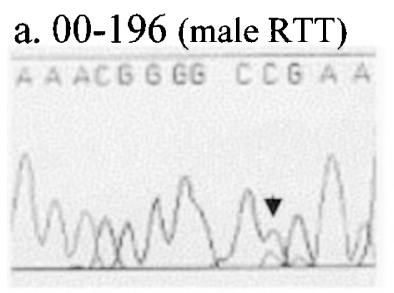

b. 99-104 (female RTT)

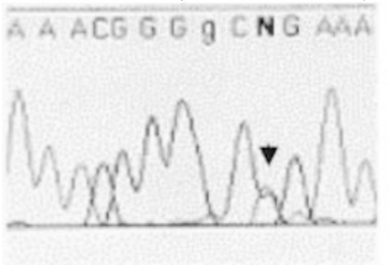

B

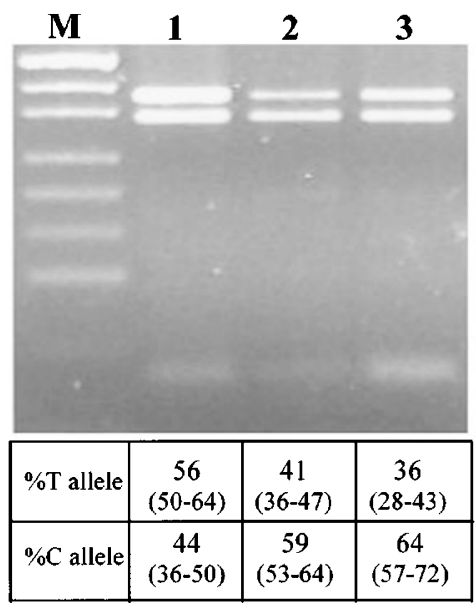

C

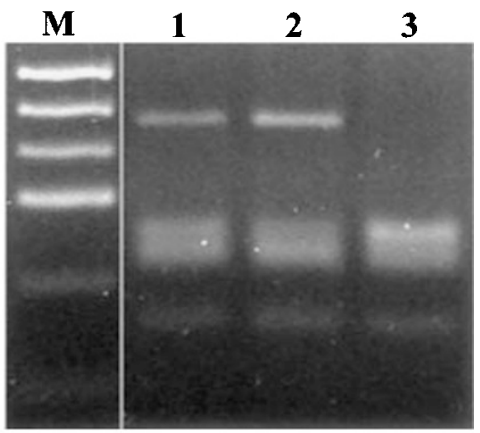

Figure 2 Analysis of the recurrent MECP2 mutation R270X. (A) Sequence analysis of patients 00-196 (BF), and 99-104 (female RTT) who are both heterozygous for the mutation R270X $(808 \mathrm{C} \rightarrow \mathrm{T})$. Recognition site of the restriction enzyme NlalV is abolished in the presence of the mutation indicated by an arrow. (B) Restriction digestion of samples 99-104 (lane 1), 00-196hr (hair root) (lane 2), and $00-196$ (lane 3) with NlalV after amplification with the first set of primers results in a pattern with fragments 370 bp (mutant allele) and $304 \mathrm{bp}+66 \mathrm{bp}$ (normal allele) in length, indicating presence of the mutant and wild type alleles for the R270X mutation in the patient samples. Results of the densitometric scans for the wild type alleles and the mutant allele in samples $00-196$ and $99-104$ is indicated below the individual lanes. The values represent the means of three independent experiments, with ranges in parenthesis. Calculations were done using the Multi-Analyst software version 1.1 (Bio-Rad Laboratories). (C) Restriction digestion of the samples $00-196$ (lane 1 ), 99-104 (lane 2), and a female control 99-91 (lane 3) with NlalV after amplification with the second set of primers results in a pattern with fragments 177, 80, 74, $49 \mathrm{bp}$ (mutant allele) and 91, 86, 80, 74, and $49 \mathrm{bp}$ (normal allele) in length, indicating presence of the mutant and wild type alleles for the R270X mutation in the patient samples. M stands for the size marker pUC Mix Marker, 8 (fragments of $501 / 489,404,331,242,190,147$, and 111/110 bp are shown in B, and fragments of 242, 190, 147, 111/110, and 67 bp are shown in C). 
then subjected to restriction endonuclease treatment since the mutation abolishes an NlaIV restriction site. Densitometric scanning of the restriction fragments revealed that the $\mathrm{T}: \mathrm{C}$ allele ratio is approximately 56:44 in the girl and approximately 36:64 in the boy (Figure 2B). Furthermore, hair root samples of BF was obtained for DNA isolation. Three independent samples were subjected to PCR amplification and restriction endonuclease treatment as indicated above. The results of these experiments also revealed the presence of somatic mosaicism with the T: C allele ration of $41: 59$. Since the restriction digestion method described above relies on the absence of digestion of the mutated allele, but does not include a control for complete digestion of the DNA, we amplified a new 380 bp fragment, which contains four constant NlaIV sites in addition to the sequences encompassing the R270X mutation. Digestion of the PCR products obtained from BF's DNA sample, and the positive control sample revealed the presence of a mutation specific $177 \mathrm{bp}$ fragment in addition to the constant fragments of $91,86,80$, 74 , and $49 \mathrm{bp}$ (Figure 2C). The $177 \mathrm{bp}$ fragment was absent in the normal control sample. This result confirmed the presence of the R270X mutation in $\mathrm{BF}$ and the positive control sample. Chromosome analysis on blood revealed a normal 46,XY karyotype. These results suggest that the boy is a somatic mosaic for the R270X mutation.

\section{Discussion}

So far, MECP2 mutations have been shown to be associated with Rett syndrome in females, predominantly as de novo mutations on the paternal X-chromosome; ${ }^{15}$ neonatal encephalopathy ${ }^{6}$ or X-linked non-specific mental retardation $^{7}$ in males which is transmitted through the maternal Xchromosome; X-linked non-specific mental retardation in females; ${ }^{7}$ and finally Rett syndrome in XY males when the mutation is present in cells alongside a normal cell line, ${ }^{8}$ or in XXY males.

Somatic mosaicism for the R270X mutation in BF could be the result of an early post-zygotic mutation or chimerism. We think chimerism - which is defined as the coexistence of different cell populations of more than one zygote in one body - is less likely since whole body chimeras result from fertilisation of one or more egg nuclei by two sperms, and is often associated with 46,XY/46,XX karyotype. ${ }^{16}$ BF's karyotype analysis did not show a chromosomal abnormality. Analysis of highly polymorphic markers used in DNA profiling for individual identification would provide the definitive answer for the post-zygotic mutation $v s$ chimerism issue since one would expect to see the inheritance of two alleles of a locus from one parent in addition to the allele from the other parent if enough loci are studied in a chimeric individual.

Based on the function of the MECP2 gene abnormal epigenetic regulation has been proposed as the mechanism underlying the pathogenesis of RTT. ${ }^{4}$ DNA methylation- dependent silencing which is a known mechanism for maintenance of X-chromosome inactivation (XCI) and gametic imprinting are good candidates to study for abnormalities in individuals with RTT. $^{6}$ In this model, escape from XCI causing biallelic overexpression of X-linked genes and/or misexpression of imprinted genes is expected. However, with the observation of a male RTT patient who is mosaic for a MECP2 mutation, disturbances in the expression of genes subject to XCI as a causative mechanism for RTT becomes unlikely since males are not subject to Xinactivation yet they display the RTT phenotype.

Somatic mosaicism has been documented in various genetic diseases including $\mathrm{X}$-linked disorders in both males and females. These include ornithine transcarbamylase deficiency, ${ }^{17}$ Duchenne muscular dystrophy, ${ }^{18}$ and hemophilia A. ${ }^{19}$ Although it is not clear whether the single-gene mutation occurred as a postzygotic event or at the halfchromatid stage, before fertilisation, ${ }^{20}$ somatic mosaicism should be considered when an X-linked dominant disease is observed in a male.

\section{Acknowledgments}

We would like to express our gratitude to the family for their help with this project. We thank Dr Uta Francke for critical reading of the manuscript, Drs Ergül Tunçbilek and Dilek Aktaş for performing the cytogenetic analysis, Dr Göknur Haliloğu for help in obtaining patient samples, and Emre Sayan, Tolga Çağatay, Tülay Arayıcı for dosage analysis experiments and DNA sequencing. This work was supported by Bilkent University, and TÜBITAK.

\section{References}

1 Rett A: Uber ein eigenartiges hirnatrophisches Syndrom bei Hyperammonemie im Kindesalter. Wien Med Wochenschr 1966; 116: $723-738$.

2 Hagberg B, Aicardi J, Dias K, Ramos O: A progressive syndrome of autism, dementia, ataxia, and loss of purposeful hand use in girls: Rett's syndrome: report of 35 cases. Ann Neurol 1983; 14: $471-479$.

3 Schanen NC and Francke U: A severely affected male born into a Rett syndrome kindred supports X-linked inheritance and allows extension of the exclusion map. Am J Hum Genet 1998; 63: $267-269$.

4 Amir RE, Van den Veyver IB, Wan M et al: Rett syndrome is caused by mutations in X-linked MECP2, encoding methylCpG-binding protein 2. Nat Genet 1999; 23: 185-188.

5 Trevathan E, Naidu S: The clinical recognition and differential diagnosis of Rett syndrome. J Child Neurol 1988; 3 (Suppl): S6S16.

6 Wan M, Sung Jae Lee S, Zhang X et al: Rett Syndrome and Beyond: Recurrent Spontaneous and Familial MECP2 Mutations at CpG Hotspots. Am J Hum Genet 1999; 65: 1520-1529.

7 Meloni I, Bruttini M, Longo I et al: A mutation in the Rett syndrome gene, MECP2, causes X-linked mental retardation and progressive spasticity in males. Am J Hum Genet 2000; 67: $982-985$

8 Clayton-Smith J, Watson P, Ramsden S, Black GCM: Somatic mutation in MECP2 as a non-fatal neurodevelopmental disorder in males. Lancet 2000; 356: 830-832. 
9 Watson P, Black G, Ramsden S et al: Angelman syndrome phenotype associated with mutations in MECP2, a gene encoding a methyl CpG binding protein. J Med Genet 2001; 38: $224-228$.

10 Philippart M: The Rett syndrome in males. Brain Dev 1990; 12 : $33-36$.

11 Eeg-Olofsson O, Al-Zuhair AGH, Teebi AS et al: A boy with Rett syndrome? Brain Dev 1990; 12: 529 - 532.

12 Topçu M, Topaloglu H, Renda Y et al: The Rett syndrome in males. Brain Dev 1991; 13: 62.

13 Hoffbuhr K, Devaney JM, LaFleur B et al: MeCP2 mutations in children with and without the phenotype of Rett syndrome. Neurology 2001; 56: 1489-1495.

14 Akyerli C, Sayi A, Kocoglu RS, Cimbis M, Topcu M, Ozcelik T: Analysis of MECP2 gene mutations in Turkish Rett syndrome patients. Eur J Hum Genet 2001; 9 (suppl. 1): P1450.

15 Trappe R, Laccone F, Cobilanschi J et al: MECP2 mutations in sporadic cases of Rett syndrome are almost exclusively of paternal origin. Am J Hum Genet 2001; 68: 1093 - 1101.
16 Repas-Humpe LM, Humpe A, Lynen R et al: A dispermic chimerism in a 2-year-old Caucasian boy. Ann Hematol 1999; 78: $431-434$.

17 Maddalena A, Sosnoski DM, Berry GT, Nussbaum RL: Mosaicism for an intragenic deletion in a boy with mild ornithine transcarbamylase deficiency. New Engl J Med 1988; 319: 999 1003.

18 Bakker E, Veenema H, Den Dunnen JT et al: Germinal mosaicism increases the recurrence risk for "new" Duchenne muscular dystropy mutations. J Med Genet 1989; 26: 553-559.

19 Leuer M, Oldenburg J, Levergne J-M et al: Somatic mosaicism in hemophilia: A fairly common event. Am J Hum Genet 2001; 69: $75-87$.

20 Gartler SM, Francke U: Half Chromatid Mutations: Transmission in Humans? Am J Hum Genet 1975; 27: 218-223.

21 Kerr AM, Nomura Y, Armstrong D et al: Guidelines for reporting different clinical features in cases with MECP2 mutations. Brain Dev 2001; 23: 208-211. 